\title{
Using the New Developed Equation to Reproduce the Enthalpies of Transfer of Urea from Water to Aqueous Ethanol, Propan-1-ol and Acetonitrile at $298 \mathrm{~K}$
}

\author{
G. Rezaei Behbehani, ${ }^{*}$ E. Tazikeh, and A. A. Saboury ${ }^{\dagger}$ \\ Department of Chemistry, Imam Khomeini International University, Qazvin, Iran. *E-mail: rezaeib@ikiu.ac.ir \\ ${ }^{\dagger}$ Institute of Biochemistry and Biophysics, University of Tehran, Tehran, Iran \\ Received September 27, 2005
}

\begin{abstract}
The enthalpies of transfer, $\Delta H_{t}^{\theta}$, of urea from water to aqueous ethanol, EtOH, propan-1-ol, $\mathrm{PrOH}$, and acetonitrile, $\mathrm{MeCN}$, are reported and analysed in terms of the new solvation theory. The analyses show that the solvation of urea is random in the considered solvent mixtures. It is also found that urea interact more strongly with $\mathrm{EtOH}$ or $\mathrm{PrOH}$ than water.
\end{abstract}

Key Words : Micro calorimeter, Preferential solvation, Urea, Variable $(\alpha n+\beta N)$

\section{Introduction}

Extension of the simple coordination model to take account of the effects of changes in solvent-solvent interactions is relatively straightforward and has been described in detail elsewhere. ${ }^{1,2}$ Briefly a solute occupies a cavity in the solvent structure, surrounded by its coordination sphere of $n$ solvent molecules. In order to complex to the solute each of these $n$ molecules will have broken some fraction, $\alpha$, of their bonds to other solvent molecules, resulting in endothermic enthalpy change of $-n \beta \Delta \Delta H^{0 *}$ where $\Delta \Delta H^{0 *}$ associated with the broken bonds. Additionally there may be a modification of solvent-solvent bonds around the coordination sphere, affecting $N$ (note $N \geq n$ ) solvent molecules. By postulating that the resulting enthalpy changes is proportional to $\Delta \Delta H^{0 *}$ we can set it equal to an enthalpy change, $-n \beta \Delta \Delta H^{0 *}$, where $\beta$ is the average proportionality constant for the modified bonds and is negative if the bonds are strengthened (leading to an exothermic contribution to the enthalpy of solution). Finally the solute may be supposed to interact with the modified solvent giving rise to an enthalpy change $\Delta \Delta H_{12}^{\theta}$. After introducing the approximation that values of $\alpha$ and $\beta$ are constant over a range of solvent compositions, and some manipulation this leads to:

$$
\begin{aligned}
\Delta H_{t}^{\theta}= & \left(\frac{p x_{B}}{x_{A}+p x_{B}}\right)\left[\Delta \Delta H_{12}+(\alpha n+\beta N) \Delta \Delta H^{\circ *}\right] \\
& -\frac{(\alpha n+\beta N)}{x_{A}+p x_{B}}\left(x_{A} L_{A}+p x_{B} L_{B}\right)
\end{aligned}
$$

where $\Delta H_{t}^{\theta}$ is enthalpy of transfer of the solute from pure solvent $A$ to mixtures of $A$ and a second solvent $B . x_{A}$ and $x_{B}$ represent the mole fractions of the components, $A$ and $B$, of the mixed solvent and $n_{A}$ and $n_{B}, N_{A}$ and $N_{B}$ are the number of $A$ and $B$ components which are the nearest neighbours of the solute. $L_{A}$ and $L_{B}$ are the relative partial molar enthalpies for a binary mixtures of $A$ and $B$ components calculated from mixing enthalpies of solvent $A$ and $B, \Delta H^{E}$, as follow:

$$
L_{A}=\Delta H^{E}+x_{B}\left(\frac{\partial \Delta H^{E}}{\partial x_{B}}\right), \quad L_{B}=\Delta H^{E}-x_{A}\left(\frac{\partial \Delta H^{E}}{\partial x_{B}}\right)
$$

$\Delta \Delta H^{0 *}$ is the difference between the $A-A$ and $B-B$ interactions in the two pure solvents and is taken as the difference between the enthalpies of condensation of the pure components. $\Delta \Delta H_{12}^{\theta}$ is the difference between the solute-B and solute-A interactions in the pure solvents, and if it is positive the solute has weaker interaction with solvent $B$ and the negative value of this parameter indicates stronger interaction of the solute with solvent $B$. The parameter $(\alpha N+\beta N)$ reflects the net effect of the solute on the solvent-solvent bonding with $\alpha N$ resulting from the formation of a cavity wherein $n$ solvent molecules become the nearest neighbours of the solute and $\beta N$ reflecting the enthalpy change from strengthening or weakening of solvent-solvent bonds of $N$ solvent molecules $(N \geq n)$ around the cavity $(\beta<0$ indicates a net strengthening of solvent-solvent bonds). The superscript $\theta$ in all cases refers to the quantities in infinite dilution of the solute. $p<1$ or $p>1$ indicate a preference for solvent $A$ or $B$ respectively; $p=1$ indicates random solvation. Analyses of $\Delta H_{t}^{\theta}$ for TBA and a series of tetraalkylammonium halides in mixed aqueous solvents revealed that there was a distinct break in the solvating properties of the mixed solvents. Thus the $\Delta H_{t}^{\theta}$ values could not be reproduced quantitatively by equation 1 across the whole range of solvent compositions. This break in the solvating properties was not found for simple electrolytes or non-electrolytes in mixed non-aqueous systems and $\Delta H_{t}^{\theta}$ could be reproduced quantitatively by equation 1 over the entire range of solvent compositions. $^{3-6}$

\section{Experimental Section}

$\mathrm{EtOH}, \mathrm{PrOH}$ and $\mathrm{MeCN}^{7-9}$ were purified as described previously. Enthalpy measurements were carried out with a four-channel commercial microcalorimeter (Thermal Activity Monitor 2277, Thermometric, Sweden). Each channel is a twin heat conduction calorimeter where the heat-flow sensor is a semi conducting thermopile (multi-junction thermocouple plates) positioned between the vessel holders and the surrounding heat sink. The insertion vessel was made from stain less steel. Urea solution $(0.1 \mathrm{mM})$ was injected by use 
Table 1. Enthalpies of transfer of urea from water to aqueous $\mathrm{EtOH}, \mathrm{PrOH}$ and $\mathrm{MeCN}$ mixtures at $25^{\circ} \mathrm{C}$ in $\mathrm{kJ} / \mathrm{mol}$

\begin{tabular}{llll}
\hline$x_{B}$ & EtOH & PrOH & $\mathrm{MeCN}$ \\
\hline 0 & 0 & 0 & 0 \\
0.11 & 0.05 & 0.06 & 0.03 \\
0.12 & 0.09 & 0.10 & 0.05 \\
0.14 & 0.14 & 0.14 & 0.07 \\
0.15 & 0.20 & 0.20 & 0.09 \\
0.16 & 0.26 & 0.25 & 0.12 \\
0.18 & 0.32 & 0.30 & 0.15 \\
0.21 & 0.38 & 0.35 & 0.19 \\
0.24 & 0.44 & 0.40 & 0.22 \\
0.28 & 0.49 & 0.45 & 0.26 \\
0.35 & 0.54 & 0.50 & 0.30 \\
0.44 & 0.59 & 0.54 & 0.34 \\
0.61 & 0.64 & 0.58 & 0.38 \\
1.00 & 0.1 & 1.55 & -0.1 \\
\hline
\end{tabular}

of a Hamilton syringe into the calorimetric stirred titration vessel, which contained $1.3 \mathrm{~mL}$ pure $\mathrm{EtOH}$, $\mathrm{PrOH}$ or $\mathrm{MeCN}$. The injection of urea solution into these pure solvents was repeated 13 times, and each injection included $0.2 \mathrm{~mL}$ of urea solution. Results are the enthalpies of solution for ternary solvent mixtures including urea in aqueous $\mathrm{EtOH}$, PrOH or $\mathrm{MeCN}$. The mixing enthalpies for aqueous $\mathrm{EtOH}, \mathrm{PrOH}$ or $\mathrm{MeCN}, \Delta H^{E}$, were measured by injection of $0.2 \mathrm{~mL}$ water into $1.3 \mathrm{~mL}$ pure $\mathrm{EtOH}, \mathrm{PrOH}$ or $\mathrm{MeCN}$ for 13 times. Enthalpies of solution of urea in solvent mixtures, $\Delta H_{S}$, were calculated by subtracting $\Delta H^{E}$ from those of for ternary mixed solvents. The enthalpies of transfer of urea, $\Delta H_{t}^{\theta}$, from water to aqueous $\mathrm{EtOH}, \mathrm{PrOH}$

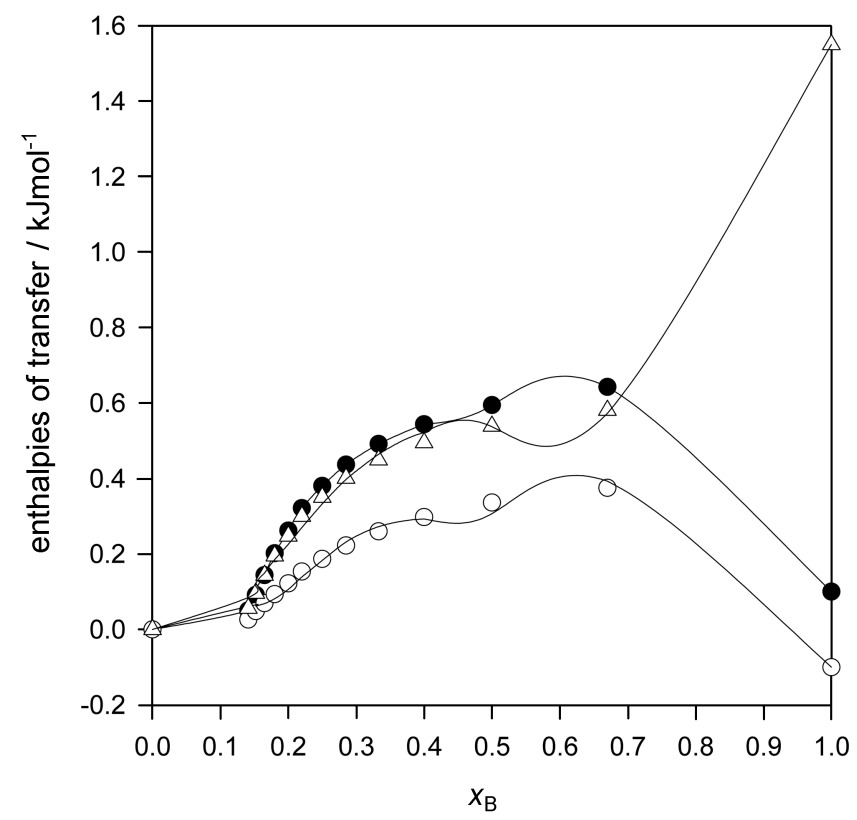

Figure 1. Comparison of the experimental (symbols) and calculated (lines) enthalpies of transfer for urea in aqueous ethanol ( - ), 1-propanol $(\triangle)$ and acetonitrile $(\bigcirc)$ via equation 9. $x_{B}$ is the mole fraction of $\mathrm{EtOH}, \mathrm{PrOH}$ or $\mathrm{MeCN}$. and $\mathrm{MeCN}$ were calculated from the enthalpies of solution, $\Delta H_{S}$, as follow:

$$
\Delta H_{t}^{\theta}=\left(\Delta H_{S}\right)_{A B}-\left(\Delta H_{S}\right)_{A}
$$

Where $\left(\Delta H_{S}\right)_{A B}$ is the enthalpy of solution of urea in aqueous EtOH, PrOH or MeCN, and $\left(\Delta H_{S}\right)_{A}$ is the enthalpies of solution of urea in pure water. $\Delta H_{t}^{\theta}$ were listed in Table 1 (in $\mathrm{kJ} / \mathrm{mol}$ ) and shown graphically in Figure 1.

\section{Discussion}

As the $\Delta H_{t}^{\theta}$ values could not be reproduced quantitatively by equation 1 over the entire range of solvent compositions, we have extended this equation. One goal of the development of the previous solvation model, is the prediction of the thermodynamics consequences of changes in the solvent system. A second approach is to use the extended equation analytically, to obtain information about the fundamental solvation process.

In the case of random solvation $(p=1)$, equation 1 simplifies to:

$\Delta H_{t}^{\theta}=x_{B}\left[\Delta \Delta H_{12}^{\theta}+(\alpha n+\beta N) \Delta \Delta H^{\circ *}\right]-(\alpha n+\beta N) \Delta H^{E}$

The enthalpy of transfer from pure solvent $A$ to pure solvent

$\mathrm{B},{ }^{A} \vec{\Delta}^{B} H_{t}^{\theta}$, is simply:

$$
{ }^{A} \vec{\Delta}^{B} H_{t}^{\theta}=\left[\Delta \Delta H_{12}^{\theta}+(\alpha n+\beta N) \Delta \Delta H^{\circ *}\right]
$$

So that equation 2 rearranges to:

$$
\frac{\Delta H_{t}^{\theta}-x_{B}{ }^{A} \vec{\Delta}^{B} H_{t}^{\theta}}{\Delta H^{E}}=(\alpha n+\beta N)
$$

As $(\alpha n+\beta N)$ is not constant over the range of solvent compositions, it is possible to change equation 4 to:

$$
\frac{\Delta H_{t}^{\theta}-x_{B}{ }^{A} \vec{\Delta}^{B} H_{t}^{\theta}}{\Delta H^{E}}=(\alpha n+\beta N)^{m i x}
$$

If the solvation is random, it is possible to define the net effect of the solute on solvent-solvent bonds in mixture, $(\alpha n+\beta N)^{m i x}$, as a combination of these values in water-rich domain, $(\alpha n+\beta N)_{A}^{\theta}$, and alcohol-rich domain, $(\alpha n+\beta N)_{B}^{\theta}$, which can be written:

$$
(\alpha n+\beta N)^{m i x}=(\alpha n+\beta N)_{A}^{\theta} x_{A}+(\alpha n+\beta N)_{B}^{\theta} x_{B}
$$

Comparing equations 5 and 6 leads to:

$$
\frac{\Delta H_{t}^{\theta}-x_{B}{ }^{A} \vec{\Delta}^{B} H_{t}^{\theta}}{\Delta H^{E}}=(\alpha n+\beta N)_{A}^{\theta} x_{A}+(\alpha n+\beta N)_{B}^{\theta} x_{B}(7)
$$

After reorganizing, leads to:

$$
\begin{aligned}
\Delta H_{t}^{\theta}= & x_{B}{ }^{A} \vec{\Delta}^{B} H_{t}^{\theta}+(\alpha n+\beta N)_{A}^{\theta} x_{A} \Delta H^{E} \\
& -x_{B}\left\lfloor(\alpha n+\beta N)_{B}^{\theta}-(\alpha n+\beta N)_{A}^{\theta}\right\rfloor \Delta H^{E}
\end{aligned}
$$

$\Delta H^{E}$ for non-random solvation is $x_{A}{ }^{\prime} L_{A}+x_{B}{ }^{\prime} L_{B}$ where 
$x_{A}{ }^{\prime}$ and $x_{B}{ }^{\prime}$ are the mole fractions of the solvent $A$ and $B$ in the solvation shell respectively. If we apply non-random conditions to equation 8 ,

$$
\begin{aligned}
& \Delta H_{t}^{\theta}=x_{B}{ }^{A} \vec{\Delta}^{B} H_{t}^{\theta}-(\alpha n+\beta N)_{A}^{\theta}\left[x_{A}{ }^{\prime} L_{A}+x_{B}{ }^{\prime} L_{B}\right] \\
& -x_{B}{ }^{\prime}\left[(\alpha n+\beta N)_{B}^{\theta}-(\alpha n+\beta N)_{A}^{\theta}\right]\left[x_{A}{ }^{\prime} L_{A}+x_{B}{ }^{\prime} L_{B}\right]
\end{aligned}
$$

Where

$$
x_{A}{ }^{\prime}=\frac{1}{x_{A}+p x_{B}}, \quad x_{B}{ }^{\prime}=\frac{p x_{B}}{x_{A}+p x_{B}}
$$

Where $(\alpha n+\beta N)_{A}^{\theta}$ and $(\alpha n+\beta N)_{B}^{\theta}$ are the net effect of the solute on solvent-solvent bonds in water-rich region and alcohol-rich region respectively. The enthalpy of transfer from pure solvent $A$ to pure solvent $B,{ }^{A} \vec{\Delta}^{B} H_{t}^{\theta}$, in equation 9 is:

$$
{ }^{A} \vec{\Delta}^{B} H_{t}^{\theta}=\Delta \Delta H_{12}^{\theta}+(\alpha n+\beta N)_{B}^{\theta} \Delta H_{B}^{\circ *}-(\alpha n+\beta N)_{A}^{\theta} \Delta H_{A}^{\circ *}
$$

$\Delta H_{A}^{\circ *}$ and $\Delta H_{B}^{\circ *}$ are the enthalpies of condensation for pure solvent $A$ and $B$ respectively. Applying equal value for $(\alpha n+\beta N)_{A}^{\theta}$ and $(\alpha n+\beta N)_{B}^{\theta}$ in equation 9 leads to equation 1 .

Equation 9 has been shown to reproduce $\Delta H_{t}^{\theta}$ for both electrolytes and non-electrolytes in a wide range of mixed aqueous and non-aqueous solvents. ${ }^{10-12}$ Using equation 9 reproducing the enthalpies of transfer shows excellent agreement between the experimental and calculated data (Figure 1) over the whole range of solvent compositions for urea in aqueous EtOH, $\mathrm{PrOH}$ and $\mathrm{MeCN}$. Solvation parameters recovered via equation 9 were reported in Table 2 . In the all cases, $(\alpha n+\beta N)_{A}^{\theta}$ values are negative, indicating that the net effect of urea is a strengthening of solvent-solvent bonds in water-rich domains.

It is generally accepted that, at low concentration, the shorter-chain alcohols, and other small organic molecules, enhance the structure of water. ${ }^{12-16}$ In aqueous alcohols $(\alpha n+\beta N)_{A}^{\theta}$ values decrease from aqueous ethanol to propan-1-ol, indicating that the enhancement of water structure by propan-1-ol is more than that of by ethanol. Thus we can conclude that at very low alcohol concentrations alcohol monomers are present in an aqueous structure which has been rigidified by interactions with alcohol alkyl residue. This is supported by results from NMR measurements of relaxation times of water and organic molecules in the mixtures, ${ }^{17}$ Xe NMR chemical shifts, ${ }^{18}$ neutron scattering, ${ }^{19}$ and dielectric relaxation. ${ }^{16}$ The positive values of

Table 2. Solvation parameters for urea in mixtures of water with $\mathrm{EtOH}, \mathrm{PrOH}$ and $\mathrm{MeCN}$ via equation 9. $\Delta \Delta H_{12}^{\theta}>0$ indicates stronger interaction of urea with water

\begin{tabular}{lcccc}
\hline Solvent system & $p$ & $(\alpha n+\beta N)_{A}^{\theta}$ & $(\alpha n+\beta N)_{B}^{\theta}$ & $\Delta \Delta H_{12}^{\theta}(\mathrm{kJ})$ \\
\hline $\mathrm{H}_{2} \mathrm{O}-\mathrm{EtOH}$ & 1 & -0.39 & 3.13 & 137.21 \\
$\mathrm{H}_{2} \mathrm{O}-\mathrm{PrOH}$ & 1 & -0.64 & 2.73 & 142.04 \\
$\mathrm{H}_{2} \mathrm{O}-\mathrm{MeCN}$ & 1 & -0.11 & -0.60 & -13.70 \\
\hline
\end{tabular}

$(\alpha n+\beta N)_{B}^{\theta}$ indicates disruption of the alcoholic structure by urea in the alcohol-rich region. The $(\alpha n+\beta N)_{B}^{\theta}$ value for aqueous acetonitrile is negative, indicating that urea strengthen the acetonitrile structure. Both $(\alpha n+\beta N)_{A}^{\theta}$ and $(\alpha n+\beta N)_{B}^{\theta}$ values for water-acetonirile mixtures is negative, indicating that the mobilities of both components decreases in the mixtures. This is in agreement with the diffusion coefficients of water and acetonitrile in wateracetonirile mixtures. ${ }^{20} p$ value for urea in aqueous EtOH, $\mathrm{PrOH}$ and $\mathrm{MeCN}$ is one, indicating that solvation of urea in these solvent mixtures is random. $\Delta \Delta H_{12}^{\theta}<0$ indicates weaker interaction of urea with water.

\section{Conclusion}

Using equation 9 reproducing the enthalpies of transfer of urea from water to aqueous ethanol, propan-1-ol and acetonitrile shows excellent agreement between the experimental and calculated data (Figure 1) over the whole range of solvent compositions which is a good support for this equation. It is clear, from these few examples and the previous published cases, ${ }^{10,11}$ that analysis of the enthalpies of transfer in this way can give remarkable insights into solvation in mixed solvents. Comparing these with the results of studies using other techniques supports this predictive theory.

\section{References}

1. Rezaei Behbehani, G.; Dillon, M.; Smyth, J.; Waghorne, W. E. J. Solution Chem. 2002, 31, 827.

2. Rezaei Behbehani, G.; Dunnion, D.; Falvey, P.; Hickey, K.; Meade, M.; McCarthy, Y.; Symons, M. C. R.; Waghorne, W. E. J. Solution Chem. 2000, 29, 521.

3. de Valera, E.; Feakins, D.; Waghorne, W. E. J. Chem. Soc. Faraday Trans. 1983, 79, 1061.

4. Cox, B. G.; Waghorne, W. E. J. Chem. Soc., Faraday Trans. 1984, $80,1267$.

5. Carthy, G.; Feakins, D.; Waghorne, W. E. J. Chem. Soc., Faraday Trans. 1987, 83, 2585.

6. Meade, M.; Waghorne, W. E.; Symons, M. C. R. J. Chem. Soc., Faraday Trans. 1996, 92, 4395.

7. Meade, M.; Hickey, K.; McCarthy, Y.; Waghorne, W. E.; Symons, M. C. R. J. Chem. Soc., Faraday Trans. 1997, 93, 536.

8. O'Duinn, C.; Feakins, D.; Waghorne, W. E. J. Solution Chem. 1986, 207, 177

9. Clune, T. G.; Cox, B. G.; Ward, A. J. I.; Waghorne, W. E. J. Chem. Soc., Faraday Trans. 1980, 76, 1131.

10. Rezaei Behbehani, G. J. Bull. Korean Chem. Soc. 2005, 2, 285.

11. Rezaei Behbehani, G. Acta Chimica Slov. 2005, 52, 288.

12. Franks, F.; Desnoyers, J. Water Sci. Rev. 1985, 1, 170.

13. Goldammer, E. V.; Hertz, H. G. J. Phys. Chem. 1970, 74, 3734.

14. Miller, K. W.; Reo, N. V.; Schoot Uiterkamp, A. J. M.; Stengle, D. P.; Stengle, T. R.; Williamson, K. L. Proc. Natl. Acad. Sci. USA 1981, 78, 4946.

15. Stengle, T. R.; Hosseini, S. M.; Basiri, H. G.; Williamson, K. L. J. Solution Chem. 1984, 13, 779.

16. Hallenga, K.; Grigera, J. R.; Beresen, J. C. J. Phys. Chem. 1980 , $84,2381$.

17. Goldammer, E. V.; Hertz, H. G. J. Phys. Chem. 1970, 74, 3734.

18. Ripmeester, J. A.; Davidson, D. W. J. Mol. Struct. 1981, 75, 67.

19. Frank, F.; Ravenhill, J.; Egelstaff, P. A.; Page, D. I. Proc. R. Soc. London, Ser. A 1970, 319, 189.

20. Easteal, A. J. Aust. J. Chem. 1980, 33, 1667. 